\title{
La Prueba Saber de Ciencias Sociales en Colombia y su utilidad pedagógica para los maestros
}

The Knowledge Test of Social Sciences in Colombia
and its pedagogical usefulness for teachers

Nancy Palacios Mena

Universidad de los Andes, Colombia

Resumen: El objetivo del artículo es analizar la naturaleza y las características del examen que evalúa el conocimiento de las ciencias sociales en secundaria en Colombia. La metodología utilizada es el análisis documental. La lectura de los documentos fue registrada en tarjetas con información como título, carácter del texto, procedencia, fecha de producción, contenido y orientación. Se pudo establecer que de un examen centrado principalmente en la memorización de contenidos, se pasó a uno en el que es importante el desarrollo de competencias, sin embargo hay críticas respecto a que este se orienta a habilidades y conocimientos evaluados en el Informe PISA, o en los que tienen interés organismos multilaterales, que a los ojos de muchos pretenden afianzar el modelo neoliberal y con ello profundizar las condiciones de pobreza y desigualdad.

Palabras clave: competencias, enseñanza secundaria, formación ciudadana, evaluación educativa, pruebas estandarizadas.

Abstract: The aim of the article is to analyze the nature and characteristics of the exam that evaluates the knowledge of the social sciences in secondary schools in Colombia. The methodology used is documentary analysis. The reading of the documents was recorded on cards with information such as title, character of the text, source, date of production, content and orientation. It was possible to establish that from an examination focused mainly on the memorization of contents, it was a transition toward one in which the development of competences is important, however there are criticisms that it is oriented to skills and knowledge evaluated in the PISA Report, or In which multilateral organizations have an interest, which in the eyes of many seek to strengthen the neoliberal model and thereby deepen the conditions of poverty and inequality.

Keywords: Competences, secondary education, citizenship training, educational evaluation, standardized tests.

(Fecha de recepción: octubre, 2017, y de aceptación: diciembre, 2017)

DOI: 10.7203/DCES.33.10833 


\section{Introducción}

Este texto forma parte de los productos de una investigación sobre la enseñanza de las ciencias sociales en la escuela secundaria en Colombia, tomando como referente empírico tres instituciones educativas del municipio de Ibagué, en el departamento del Tolima - Colombia. Las quejas frecuentes de los profesores de las universidades del país sobre el bajo nivel de los estudiantes al finalizar la secundaria, las controversias entre los maestros de primaria y de secundaria por la adopción de la educación por competencias, la resistencia derivada de las críticas que se le hacen a dicho enfoque y la prevalencia de modelos de enseñanza basados en la memorización y la repetición de contenidos con escasa comprensión, plantean la necesidad de indagar sobre los niveles de progresión del aprendizaje en el área de ciencias sociales.

El artículo es parte de la investigación mencionada, en la que se problematiza la relación entre la evaluación como un indicador de la calidad de la educación y las estrategias pedagógicas utilizadas en clase de ciencias sociales. Con el análisis de los resultados de las pruebas Saber 11 obtenidos por los estudiantes en esa área, se pretende establecer qué aprendizajes evidencian los resultados de las pruebas de Ciencias Sociales y Competencias Ciudadanas, y qué influencia tienen las estrategias de evaluación utilizadas en clase en los resultados de los estudiantes en las pruebas Saber 11. La pregunta de investigación que se busca responder con el artículo es: ¿qué impacto tienen las pruebas estandarizadas de ciencias sociales en el trabajo que hacen los maestros en el salón de clases? De esta forma, se pretende establecer la congruencia entre los objetivos de aprendizaje planteados en los documentos oficiales para el área de ciencias sociales, y los contenidos de esta en las Pruebas Saber. Por ello, el texto se enfoca en caracterizar las competencias evaluadas en la prueba de ciencias sociales y el uso pedagógico que le pueden dar los maestros de secundaria. Estos estudios surgen de la necesidad de fomentar investigaciones que acerquen los datos obtenidos en las pruebas de evaluación externa al trabajo cotidiano de las aulas del país, con el ánimo de dinamizar procesos para su lectura idónea, reflexiones curriculares y pedagógicas, aportes a los lineamientos orientadores de las pruebas, diálogo entre los agentes que intervienen directa o indirectamente en la organización de los sistemas de educación nacional, y todo aquello que redunde en la cualificación de las estrategias de formación y evaluación educativas y del crecimiento de la sociedad colombiana en cuanto a desarrollo del saber y del bienestar social (Rojas, 2014).

\section{Método}

La metodología utilizada es la revisión documental. Las variables que se tuvieron en cuenta son: la inclusión de la evaluación como indicador de la calidad educativa, el contexto histórico de la prueba de ciencias sociales, la evolución de los componentes evaluados en la prueba, la justificación de la fusión de las pruebas de ciencias sociales con 
competencias ciudadanas, y la relación entre las Pruebas Saber y los estándares curriculares del área. Las fuentes analizadas fueron los siguientes documentos: Sistema Nacional de Evaluación Estandarizada de la Educación; la Alineación del Examen Saber 11; la Guía de interpretación y uso de los resultados de las Pruebas Saber 11 (versión 1), el documento Examen de Estado 2011, la Guía de interpretación y uso de los resultados del examen Saber 1, Establecimientos educativos, la versión 1 del cuadernillo del examen presentado en 2014, y los resultados para estudiantes del examen de antes de 2014 y del actual.

La sistematización de los documentos tuvo dos etapas. En un primer momento se estudiaron las guías, folletos e instructivos publicados por el Instituto Colombiano para la Evaluación de la Educación (de aquí en adelante Icfes), para socializar los contenidos y los cambios que se le introducen a la prueba, y en un segundo momento se examinaron los niveles de congruencia entre lo que evalúa la prueba y los estándares básicos de competencias en ciencias sociales y formación para la ciudadanía. Con ese fin, se leyó detalladamente la prueba de ciencias sociales que los estudiantes presentaron en 2014, se observó su estructura y el uso pedagógico que puede tener.

\section{Marco teórico y antecedentes}

La realización del trabajo requirió de la revisión de dos tipos de documentos, por un lado, los documentos, instructivos y guías utilizadas por el Icfes para divulgar el contenido y la forma de interpretación de los resultados de la prueba saber 11 y por el otro, la revisión de algunos trabajos empíricos que se han ocupado de la misma problemática. Entre los trabajos producidos por el Icfes se destaca el anexo tres publicado en el año 2013 con el título La prueba de competencias de sociales y ciudadanas. El anexo en cuestión contiene los antecedentes de la prueba de ciencias sociales, la evaluación de la prueba de competencias ciudadanas, la justificación de la fusión de las dos pruebas, las competencias y los componentes que tendría la prueba a partir del año 2014 (Icfes, Ministerio de Educación, 2013). Otro documento producido por estas dos instituciones al que se tuvo acceso fue La guía de interpretación y uso de resultados de las pruebas saber 11 para establecimientos educativos, versión 1. Esta guía tiene información detallada y complementada con ejemplos sobre la manera de ingresar a consultar los resultados, la metodología utilizada para realizar la clasificación de las instituciones educativas y sus sedes, los resultados que se reportan por pruebas y subpruebas y el análisis de algunas preguntas como las que están contenidas en el examen (Icfes, Ministerio de Educación, 2015).

Un tercer referente de consulta obligado fue un libro sobre la evolución de las pruebas de estado. En la obra se analizan las intimidades de la prueba, se examinan las preguntas, y además se precisa qué se evalúa y cuál es la forma de hacerlo. El trabajo logró establecer que el currículo es el puente entre las pruebas y lo que los alumnos adquieren o deben adquirir en la escuela. Según 
lo planteado en el texto las pruebas obligan a que haya ciertos niveles de exigencia relacionados con los saberes básicos; con las pruebas se aprecia la relación entre currículo y escuela (Icfes, 2004). Adicionalmente, se consultaron algunos trabajos empíricos en el campo. Uno de ellos fue publicado en el 2014 con el título Competencias en lenguaje: relaciones entre la escuela y las pruebas de Estado. Este estudio hace parte de algunas iniciativas de investigadores financiadas por el Icfes, y tuvo como objetivos identificar las competencias del lenguaje evaluadas en cinco instituciones del Distrito Capital y establecer su relación con los resultados de la prueba Icfes-2012. El estudio permitió caracterizar los enfoques de evaluación aplicados por los docentes de la asignatura curricular de lengua castellana y literatura, a la vez que facilitó la caracterización del rendimiento académico de sus estudiantes, según la información provista por las bases de datos Saber $11^{\circ}-2010$ y los resultados de la evaluación adelantadas por la institución. Los resultados de esta investigación permitieron establecer las fortalezas y debilidades que se presentan en el desempeño de esos estudiantes y el nivel de la relación entre lo evaluado en el aula con aquello establecido por la prueba de estado en el caso específico de lenguaje (Rojas, 2014).

En un trabajo sobre la prueba de acceso a la universidad (selectividad) en España y las rutinas escolares en la enseñanza de la historia y la geografía, se analizaron los protocolos de examen y las respuestas de los alumnos. Souto, Fuster y Sáiz (2014) abordaron el nivel cognitivo solicitado a los estudiantes, tratando de identificar las competencias que desarrollan y luego intentan comprobar si la configuración de la prueba en la comunidad valenciana permite valorar la conciencia histórica de los alumnos o, por el contrario, estos siguen demostrando un conocimiento aislado. Los hallazgos de Souto et al. (2014) establecieron que las pruebas de acceso a la universidad (PAU) se realizan desde las rutinas y no desde presupuestos teóricos de innovación, esto plantea como un reto importante comprobar en qué grado el examen y como está diseñado y aplicado condiciona el discurso histórico no solo de profesores, sino también de libros de texto, y cómo éstos afectan al discurso de los estudiantes. Finalmente concluye que los estudiantes preuniversitarios necesitan una historia no únicamente para obtener éxito en la PAU sino para que les sea útil en la interpretación del presente que viven y sean capaces de dar alternativas al futuro que les espera.

Por su parte, el artículo de Vicente Pérez (2015) sobre el lugar del examen en la escuela tuvo como objetivo analizar el papel que ha jugado y juega la práctica examinatoria en las clases de sociales, geografía e historia de la enseñanza secundaria. El trabajo buscó responder entre otras preguntas: ¿qué papel juegan el examen y la evaluación tradicional como factor limitante del cambio en educación?, ¿cómo se puede influir y /o cambios pedagógicos para dar con una evaluación más formativa, menos selectiva? ¿Podemos realmente convertir la evaluación en un instrumento de aprendizaje formativo, individual y colectivo? 
Una de las principales conclusiones de la tesis es que la práctica evaluadora desempeña un papel importante en la legitimación y fabricación de subjetividades en la escuela y que uno de los obstáculos más obstinados a cualquier cambio educativo en profundidad empieza por la desnaturalización del examen escolar y termina por la impugnación de los usos y abusos que se hace de la evaluación (Pérez, 2015).

En el ámbito regional se tuvo acceso al trabajo de Pedro Ravela (2010) titulado ¿Qué pueden aportar las evaluaciones estandarizadas a la evaluación en el aula? En el marco de la investigación se realizó una indagación de carácter exploratoria y descriptiva en ocho países de América Latina sobre los enfoques de evaluación de aprendizaje en lectura y matemáticas de maestros de $6^{\circ}$ grado de primaria. El estudio se enfocó en torno a cuáles son los enfoques discursivos desde los cuales los maestros conciben sus prácticas de evaluación, qué tipo de consignas de evaluación utilizan para medir aprendizajes, cómo emplean sus evaluaciones para orientar a sus estudiantes y cómo pueden las evaluaciones estandarizadas enriquecer las prácticas de evaluación en el aula. Los hallazgos de Ravela (2010) le permitieron establecer que según los profesores participantes hay un conjunto de aspectos en los que las pruebas externas enriquecen los enfoques y prácticas de los maestros como lo son la toma de conciencia sobre contenidos a trabajar con los alumnos, la apertura a nuevos enfoques conceptuales y prácticos, el acceso a instrumentos para el trabajo en el aula, la inspiración y modelos para el diseño de materiales, el preparar a los alumnos para las mismas pruebas y el seguimiento de estudiantes individuales y de resultados grupales.

En el marco de una investigación del área del lenguaje de la Subdirección Académica del ICFES cuyo propósito era analizar los presupuestos disciplinares y pedagógicos que subyacen a la evaluación de competencias en lenguaje, Castillo (2004) produjo un artículo titulado La evaluación de competencias y sus implicaciones pedagógicas 1991 - 2004. El texto buscó demostrar cuáles eran las exigencias de la evaluación por competencias del área de lenguaje en ese periodo de acuerdo con las políticas estipuladas por el Instituto y qué se había logrado y qué faltaba por lograr en el ambiente educativo con el fin de alcanzar el ideal de una educación que pretende resignificar la interacción en el aula. Su trabajo apuntaba específicamente a comprender cuál es el nivel deseado en la formación en el lenguaje en la educación básica y media y qué resultados arroja la evaluación al terminar estos estos ciclos. Castillo (2004) concluyó que el objetivo principal de la lengua materna debe apuntar a que el estudiante use el lenguaje como herramienta fundamental de interacción con el saber. Desde esta perspectiva, una de las labores fundamentales de los docentes, sea cual sea su área trabajo, debe ser la de propiciar el acceso a mayores niveles de dominio sobre el lenguaje. "Lograr lo anterior implica implementar trabajos de lectura que amplíen el saber medio de los estudiantes en la básica y seleccionar y trabajar con 
textos que le permitan al docente y al estudiante enfrentar formas de organización textual y discursiva diversas, que exigen en el lector niveles de abstracción y análisis diferentes" (p.77).

Finalmente, para el rastreo de antecedentes se consultó el trabajo Características de la enseñanza y la evaluación de los aprendizajes en educación básica primaria (Prado y Medina, 2014). En este se aborda la reflexión de la educación desde la perspectiva del rendimiento de los estudiantes en las evaluaciones externas tipo Saber $3^{\circ} \mathrm{y}$ $5^{\circ}$, es decir, en la Educación Básica Primaria, y su rendimiento vinculado a los modelos pedagógicos o didácticos que utilizan los docentes de estos cursos en las Instituciones Educativas Distritales. Los investigadores apelaron a un estudio de carácter cualitativo con una pretensión explicativa y fundado epistémicamente en un análisis de corte hermenéutico, con la vinculación de dos grupos de Instituciones Educativas Distritales. El primero de ellos, cuenta con resultados ubicados en los rangos de mínimos e insuficientes, y el segundo, con resultados avanzados y satisfactorios. Con esta selección buscó encontrar puntos de contraste en donde divergen y convergen las prácticas educativas para estimar su potencial relación con los resultados en estas evaluaciones externas. Aunado a ello, se puede apreciar cómo a pesar de conservar un mismo marco político, se generan dinámicas pedagógicas heterogéneas en la realidad escolar, debido a la comprensión, la aplicación y la concreción de los lineamientos desde la autonomía insti- tucional y la libertad de enseñanza que cobija a la acción docente.

En este trabajo se analizan los modelos pedagógicos o didácticos, se indagó sobre los elementos conceptuales o teóricos que, según los docentes y Directivos, guían el desarrollo de su práctica, las características de las prácticas de orden didáctico, las interacciones educativas entre los agentes educativos, el clima institucional y los planes de estudio. En relación con la evaluación de los aprendizajes de los estudiantes se pretendió responder preguntas como qué es evaluación, cómo se evalúa, qué se evalúa, para qué se evalúa y cómo debería evaluarse. También se incluyó como categoría analítica las políticas educativas, buscando los elementos que pueden llegar a direccionar las prácticas de enseñanza y la evaluación de los aprendizajes directamente en las Instituciones educativas. Para ello se analizaron los planes de Desarrollo Sectorial desde el 2004 hasta la planeación prevista para 2016 y los Proyectos Educativos Institucionales PEI como carta de navegación de la institución y construidos de manera consensuada y participativa (Prado y Medina, 2014).

\section{Resultados}

Los cambios en la prueba de ciencias sociales

Hasta antes del año 2000 la prueba estaba centrada en evaluar la apropiación memorística de contenidos y, en menor medida, algunas habilidades. A partir de ese año se institucionalizó la evaluación por competencias, que exa- 
mina la apropiación de conocimientos del área, y la adquisición de habilidades y destrezas para utilizar dichas habilidades en determinados contextos. Desde la perspectiva del Icfes, la prueba tiene como finalidad apoyar los procesos de selección y admisión que hacen las instituciones de educación superior. Hay que destacar que los cambios que se le han introducido a las Pruebas Saber $11^{\circ}$ a partir del año 2000, han surgido de la necesidad de articularlas con los documentos curriculares que ha producido el Ministerio y con lineamientos, estándares y derechos básicos de aprendizaje, pero también de alinear las pruebas que se han venido implementado y que se encuentran ya institucionalizadas en $3 .^{\circ}, 5 .^{\circ}$ y $9 .^{\circ}$ y en la educación superior. Los lineamientos curriculares de ciencias sociales fueron definidos como puntos de apoyo y orientación general que se editaron con el ánimo de aportar a las y los maestros del país elementos de tipo conceptual y metodológico que dinamizaran en gran modo su quehacer pedagógico (Ministerio de Educación, 2002). Los estándares básicos de competencia de ciencias sociales fueron definidos como criterios claros y públicos que permites juzgar si un estudiante, una institución o el sistema educativo en su conjunto, cumplen con unas expectativas comunes de calidad; expresan una situación deseada en cuanto a lo que se espera que todos los estudiantes aprendan en cada una de las áreas a lo largo de su paso por la Educación Básica y Media (Ministerio de Educación, 2006).

En cuanto a la implementación de los exámenes, la prueba del núcleo común se aplicaba a todos los estudiantes, las pruebas de profundización de cada área, que tenían un mayor grado de complejidad que la del núcleo común, era optativa para los estudiantes quienes elegían el are a profundizar según sus fortalezas. Las áreas interdisciplinarias pretendían valorar competencias sociales, culturales y ambientales en las que los estudiantes relacionaran saberes de diferentes ciencias. "La prueba de ciencias sociales tenía como objetivos examinar competencias referentes a la valoración del mundo social" (Icfes, 2013, p. 7). La tabla 1 presenta la estructura de la prueba de ciencias sociales hasta 2014, en función de lo planteado en los lineamientos curricu-

\section{Tabla I}

\section{Estructura de la prueba de ciencias sociales aplicada hasta 2014}

(Fuente: Elaboración propia a partir de Icfes, Ministerio de Educación, 2011: p 8 y 9)

\begin{tabular}{|l|l|}
\hline Competencias & Componentes \\
\hline Interpretativa & El espacio, el territorio, el ambiente y la población \\
\hline Argumentativa & El poder, la economía y las organizaciones sociales \\
\hline Propositiva & El tiempo y las culturas \\
\hline
\end{tabular}


lares y los estándares de competencias del área publicados por el Ministerio de Educación.

Las competencias contempladas y enunciadas en la tabla 1 evalúan principalmente la capacidad de los alumnos para comprender la información contenida en textos de diferente naturaleza, informes periodísticos, tablas y gráficos estadísticos, mapas, planos, fragmentos de una lectura o la lectura completa. Los componentes de la disciplina evaluados responden a tres ámbitos: uno que incluye los procesos históricos y su legado, otro, el componente de la economía, la organización, la estructura del Estado y sus instituciones, y uno más que incluye el componente espacial, que prioriza la manera como las condiciones físicas determinan procesos sociales y la forma como las comunidades utilizan y modifican el espacio geográfico. Con estas competencias y estos ámbitos se pretendió abarcar los tres componentes que la Ley General de Educación (Ministerio de Educación, 1994), dicha ley definió como componentes del área de ciencias sociales: la historia, la geografía y la Constitución Política, pero también dio cabida a otros saberes como los de la antropología, la ciencia política, la sociología y la lingüística, propuestos en los lineamientos curriculares del área.

Con el objetivo de consolidar el Sistema de Evaluación Estandarizada de la Educación (SNEE) y de introducir mejoras en las pruebas en todos los niveles de educación básica, media y superior, el Ministerio de Educación Nacional y el Icfes presentaron una propuesta de alineación de las Pruebas Saber $11^{\circ}$.
Con la alineación se pasaría de un sistema con mediciones aisladas para la educación media, a uno que hiciera un seguimiento sistemático de los resultados de la educación a través de los diferentes niveles. El nuevo examen incluye cinco pruebas: lectura crítica, matemáticas, ciencias sociales y competencias ciudadanas, ciencias naturales e inglés. Estas pruebas evalúan siete áreas /competencias: lectura crítica, matemáticas, razonamiento cuantitativo, ciencias naturales, ciencias sociales, competencias ciudadanas e inglés.

En relación con la alineación de la prueba de ciencias sociales, el cambio principal fue la fusión en un solo examen de las ciencias sociales y las competencias ciudadanas, que se hizo con la premisa de que el desarrollo en las últimas décadas de las ciencias sociales ha llevado a que se incorporen dentro de su estudio elementos afines a la ciudadanía, con lo que se justifica la decisión de hacer una evaluación conjunta para las dos área. Para el Icfes, las competencias cognitivas que se requieren en ciudadanía encuentran en la formación escolar en ciencias sociales un espacio apropiado para su desarrollo. Ligado al cambio anterior, y habiendo identificado los elementos fundamentales que se deben evaluar en ciencias sociales y en competencias ciudadanas, el segundo cambio se hizo en la estructura de la prueba (ver tabla 2), en la que se definió la evaluación de tres competencias: pensamiento social, interpretación y análisis de perspectivas, y pensamiento sistémico y reflexivo. La actual estructura de la prueba ilustrada en la tabla 2, evidencia que en la determinación de las 


\section{Tabla II}

\section{Estructura actual de la prueba de ciencias sociales y competencias ciudadanas}

(Fuente: Elaboración propia a partir de Icfes, Ministerio de Educación, 2013:p. 85 y 86)

\begin{tabular}{|c|c|c|c|}
\hline Competencia & $\begin{array}{l}\text { Estándares básicos } \\
\text { de ciencias sociales } \\
\text { y de competencias } \\
\text { ciudadanas }\end{array}$ & $\begin{array}{l}\text { Definición } \\
\text { de la competencia }\end{array}$ & $\begin{array}{l}\text { Habilidades } \\
\text { que se evalúan }\end{array}$ \\
\hline $\begin{array}{l}\text { Pensamiento } \\
\text { social }\end{array}$ & $\begin{array}{l}\text { Relaciones espaciales } \\
\text { y ambientales. } \\
\text { Convivencia y paz. }\end{array}$ & $\begin{array}{l}\text { Esta competencia supo- } \\
\text { ne que la apropiación } \\
\text { de conceptos básicos es } \\
\text { el fundamento para el } \\
\text { desarrollo de un pensa- } \\
\text { miento en ciencias socia- } \\
\text { les cuyo ámbito de apli- } \\
\text { cación trascienda dichas } \\
\text { ciencias hasta llegar a } \\
\text { los ejercicios cotidianos } \\
\text { de la ciudadanía. }\end{array}$ & $\begin{array}{l}\text { Se indaga por las herra- } \\
\text { mientas de pensamiento } \\
\text { con que cuentan } \\
\text { los estudiantes para } \\
\text { reconocer, diferenciar y } \\
\text { usar conceptos básicos de } \\
\text { las ciencias sociales. }\end{array}$ \\
\hline $\begin{array}{l}\text { Interpretación } \\
\text { y análisis de } \\
\text { perspectivas }\end{array}$ & $\begin{array}{l}\text { Relaciones ético- } \\
\text { Políticas. } \\
\text { Participación y responsa- } \\
\text { bilidad democrática. }\end{array}$ & $\begin{array}{l}\text { Esta competencia se } \\
\text { refiere a la capacidad de } \\
\text { reconocer perspectivas y } \\
\text { analizarlas, } \\
\text { en particular por medio } \\
\text { del examen de los argu- } \\
\text { mentos que presente el } \\
\text { representante, individuo } \\
\text { o colectivo de determina- } \\
\text { da perspectiva o posición. }\end{array}$ & $\begin{array}{l}\text { Evalúa los usos de evi- } \\
\text { dencias en argumenta- } \\
\text { ciones y explicaciones, y } \\
\text { la solidez y pertinencia } \\
\text { de estas. La validez y } \\
\text { coherencia de enuncia- } \\
\text { dos hechos por diferentes } \\
\text { actores. La afinidad que } \\
\text { pueda existir entre dife- } \\
\text { rentes perspectivas. }\end{array}$ \\
\hline $\begin{array}{l}\text { Pensamiento } \\
\text { reflexivo y sis- } \\
\text { témico }\end{array}$ & $\begin{array}{l}\text { Relaciones con la histo- } \\
\text { ria y las culturas. } \\
\text { Pluralidad, identidad y } \\
\text { valoración de las diferen- } \\
\text { cias. }\end{array}$ & $\begin{array}{l}\text { Esta competencia se } \\
\text { entiende como la habi- } \\
\text { lidad de reconstruir y } \\
\text { comprender la realidad } \\
\text { social desde una perspec- } \\
\text { tiva sistémica, usando } \\
\text { conceptos propios de las } \\
\text { ciencias sociales. }\end{array}$ & $\begin{array}{l}\text { Se evalúa la capacidad } \\
\text { de los estudiantes para } \\
\text { identificar causas de una } \\
\text { situación problemática; } \\
\text { establecer qué tipos de } \\
\text { elementos están presen- } \\
\text { tes en ella; comprender } \\
\text { qué tipo de factores se } \\
\text { enfrentan; comprender } \\
\text { qué dimensiones se privi- } \\
\text { legian en una determina- } \\
\text { da solución; anticipar los } \\
\text { efectos de la implemen- } \\
\text { tación de una solución y } \\
\text { evaluar su aplicabilidad } \\
\text { en determinado contexto. }\end{array}$ \\
\hline
\end{tabular}


competencias que se incluyeron, su conceptualización y los criterios de evaluación, hay un claro propósito de articular los estándares básicos de competencias de ciencias sociales y ciudadanas. En la definición de las competencias son fundamentales la comprensión de fenómenos sociales, la apropiación de conceptos propios de las ciencias sociales, la aprehensión de actitudes y valores que hagan explícita la adquisición de cierto nivel de pensamiento crítico frente a la realidad cotidiana, y la apropiación de habilidades y destrezas propias de la labor de un científico social.

En lo que tiene que ver con los resultados, la actual prueba de ciencias sociales proporciona a los estudiantes el promedio que representa el desempeño medio de los estudiantes en determinada prueba, este resultado se reporta en una escala de 0 a 100 puntos, sin decimales. La desviación estándar es una medida de dispersión de los datos que indica que cuanto más alto sea este valor, más alejados del promedio se encontrarán los resultados de los estudiantes, lo cual indica mayor dispersión o heterogeneidad entre los puntajes obtenidos por ellos. Los niveles de desempeño describen lo que saben y saben hacer los estudiantes de acuerdo con las especificaciones de una determinada prueba del examen. Se establecieron cuatro niveles de desempeño: 1, 2, 3 y 4 , entre los cuales el 4 se define como el nivel esperado (Icfes, 2016). La suma de los porcentajes puede no ser exactamente $100 \%$ debido a aproximaciones decimales. Algunas preguntas de ciencias sociales contribuyen al puntaje de la subprueba de competencias ciuda- danas. Es decir, la prueba de ciencias sociales da una nota, y las preguntas de esta prueba que involucran alguno de los ámbitos de formación para la ciudadanía dan otros puntos que se presentan con una nota aparte de las ciencias sociales y formación para la ciudadanía. La descripción de los niveles de desempeño es fundamental porque, como se indicaba, describe qué saben y qué no saben hacer los estudiantes y en qué tienen fortalezas y deficiencias.

En el nuevo examen esta es una información a la que se le ha dado mucha relevancia, el reto de los establecimientos es sacar a todos sus estudiantes del rango uno y e intentar llevarlos del rango dos al tres y de este al cuatro. Los datos de los estudiantes por nivel de desempeño cumplen una función clasificatoria, y de poca utilidad pedagógica, por cuanto la información que arrojan es muy general y no es suficiente para determinar dentro de cada rango específicamente cuáles son las habilidades concretas que han desarrollado los estudiantes y cuáles no, de manera que se puedan fortalecer las debilidades y llevar a los estudiantes al siguiente rango.

\section{Discusión}

\section{La prueba de ciencias sociales hoy}

En este estudio se realizó un análisis del examen que los estudiantes de grado $11{ }^{\circ}$ presentaron en 2014 . El resultado fueron unas categorías que de manera individual y en su conjunto nos permiten establecer la estructura de la prueba y el uso pedagógico de la misma. 
Estructura de la prueba

El examen que se analizó fue presentado por los estudiantes en 2014. La prueba de ciencias sociales es la tercera de dicho examen y fue ubicada entre la prueba de lectura crítica y la de ciencias naturales. Se formularon 25 preguntas que van de la 51 a la 75. Para estudiar la estructura de la prueba organizamos las preguntas en tres grupos, siguiendo las tres competencias propuestas por el Ministerio y el Icfes. Esta clasificación presentó algunas dificultades porque, como veremos más adelante, no siempre es claro a qué competencia puede pertenecer una pregunta: podría ser a dos de ellas por la manera como se plantea la definición de la competencia, los estándares curriculares a los que apunta, y las habilidades evaluadas.

La definición de esta competencia es muy amplia, como lo muestra la tabla 3 en ella se incluyen preguntas que evalúan la dimensión espacial y temporal, que están relacionadas con los factores

Tabla III

\section{Preguntas de la competencia "pensamiento social", Pruebas Saber, ciencias sociales (2014)}

Fuente: Elaboración propia a partir de Icfes, Ministerio de Educación, 2015:p. 19-24

\begin{tabular}{|l|l|}
\hline Competencia: Pensamiento social & Número de preguntas: 7 \\
\hline Temática & Solicitud de la pregunta \\
\hline Acción de tutela. & ¿Cuándo se aplica la tutela? \\
\hline Abandono de zonas del centro de la ciudad. & Causa del fenómeno. \\
\hline Revolución Industrial. & Consecuencias en la población. \\
\hline La Constitución y el Estado de derecho. & La utilidad de la Constitución. \\
\hline La crisis de 1929. & $\begin{array}{l}\text { ¿En qué consiste la sustitución de importaciones } \\
\text { y su impacto? }\end{array}$ \\
\hline El conflicto árabe-israelí. & $\begin{array}{l}\text { La causa del no reconocimiento del Estado } \\
\text { palestino. }\end{array}$ \\
\hline El gobierno escolar de una institución educativa. & $\begin{array}{l}\text { Organización, nombramiento y funciones de los } \\
\text { órganos de gobierno escolar. }\end{array}$ \\
\hline Habilidades que evalúa & \\
\hline Identificar y usar conceptos sociales básicos. & \\
\hline Localizar en el tiempo y en el espacio eventos históricos y prácticas sociales. \\
\hline Analizar dimensiones históricas de eventos, problemáticas y fenómenos sociales. \\
\hline $\begin{array}{l}\text { Conocer el modelo de Estado social de derecho, su aplicación en Colombia, la organización del Esta- } \\
\text { do y los mecanismos de participación ciudadana. }\end{array}$ \\
\hline
\end{tabular}


que generan pobreza y desigualdad social, con la dimensión económica, política y cultural, con el sistema político y con la estructura del Estado, el aparato normativo del país y las instituciones y su funcionamiento. Según lo planteado en la definición de esta competencia (Icfes, Ministerio de Educación, 2015), lo que aglutina la gran variedad de aspectos que en ella se contemplan es

\section{Tabla IV. Preguntas de la competencia "interpretación y análisis de perspectivas", Pruebas Saber, ciencias sociales (2014)}

(Fuente: Elaboración propia a partir de Icfes, Ministerio de Educación, 2015, p. 19-24)

\begin{tabular}{|c|c|}
\hline $\begin{array}{l}\text { Competencia: Interpretación y aná- } \\
\text { lisis de perspectivas }\end{array}$ & Número de preguntas: diez \\
\hline Temática & Solicitud de la pregunta \\
\hline $\begin{array}{l}\text { Asentamiento de desplazados en un } \\
\text { humedal. }\end{array}$ & Las intenciones de las organizaciones implicadas. \\
\hline Discriminación a las mujeres. & $\begin{array}{l}\text { Actuación del profesor según lo establecido en la Cons- } \\
\text { titución. }\end{array}$ \\
\hline Derechos constitucionales. & $\begin{array}{l}\text { La coherencia de una acción que atiende un fallo judi- } \\
\text { cial. }\end{array}$ \\
\hline Derechos constitucionales. & $\begin{array}{l}\text { La identificación de acciones que producen vulneración } \\
\text { de derechos. }\end{array}$ \\
\hline El sistema político de un país. & $\begin{array}{l}\text { Relación de un discurso con un período de la historia } \\
\text { política. }\end{array}$ \\
\hline Violencia contra la mujer. & Los intereses que favorecen la toma de una posición. \\
\hline El tráfico de drogas y su legalización. & La afinidad entre un argumento y una propuesta. \\
\hline $\begin{array}{l}\text { Los contenidos de los medios de comuni- } \\
\text { cación. }\end{array}$ & $\begin{array}{l}\text { ¿Qué actitud tienen los ciudadanos frente a las noti- } \\
\text { cias? }\end{array}$ \\
\hline Discriminación por orientación sexual. & $\begin{array}{l}\text { ¿Por qué es importante desarrollar políticas de igual- } \\
\text { dad y respeto por la diferencia? }\end{array}$ \\
\hline Violencia contra la mujer. & Las consecuencias de las acciones de funcionario \\
\hline \multicolumn{2}{|l|}{ Habilidades que evalúa } \\
\hline \multicolumn{2}{|c|}{ Analizar perspectivas de distintos actores y grupos sociales. } \\
\hline \multicolumn{2}{|c|}{ Evaluar posibilidades y limitaciones del uso de fuentes. } \\
\hline \multicolumn{2}{|c|}{ Develar prejuicios e intenciones en enunciados o argumentos } \\
\hline \multicolumn{2}{|c|}{ Reconocer y comparar perspectivas de actores y grupos sociales. } \\
\hline $\begin{array}{l}\text { Establecer relaciones entre las perspect } \\
\text { propuestas de solución }\end{array}$ & de los individuos en una situación conflictiva y las \\
\hline
\end{tabular}


que supone la apropiación de conceptos básicos, y se constituye en el fundamento para el desarrollo de un pensamiento propio de las ciencias sociales, cuyo ámbito de aplicación trasciende estas ciencias hasta posibilitar ejercicios cotidianos de ciudadanía.

Las preguntas correspondientes a esta competencia, incluidas en la tabla
4 , requieren que los estudiantes, a propósito de un conflicto, estén en capacidad de comprender su origen, entender qué buscan los diferentes actores, identificar las coincidencias y diferencias entre sus intereses, y valorar la reacción (de aceptación o rechazo) de las partes ante una propuesta de solución. Según lo planteado por el (Icfes, Minis-

\section{Tabla V}

\section{Preguntas de la competencia "pensamiento reflexivo y sistémico", Pruebas Saber, ciencias sociales (2014)}

(Fuente: Elaboración propia a partir de Icfes, Ministerio de Educación, 2015: p. 19-24)

\begin{tabular}{|l|l|}
\hline $\begin{array}{l}\text { Competencia: Pensamiento reflexivo y sisté- } \\
\text { mico }\end{array}$ & Número de preguntas: 8 \\
\hline Temática de la pregunta & ¿Solicitud de la pregunta \\
\hline Liberalismo económico. & $\begin{array}{l}\text { Establecer la similitud entre un enunciado y } \\
\text { una acción. }\end{array}$ \\
\hline El socialismo. & $\begin{array}{l}\text { El concepto de socialismo y las acciones para } \\
\text { alcanzarlo. }\end{array}$ \\
\hline Programas de apoyo a jóvenes. & La pertinencia de una medida. \\
\hline $\begin{array}{l}\text { La construcción de obras de infraestructura en } \\
\text { territorios indígenas. }\end{array}$ & $\begin{array}{l}\text { La actuación del Estado en un territorio indí- } \\
\text { gena. }\end{array}$ \\
\hline $\begin{array}{l}\text { La concesión de un territorio indígena para la } \\
\text { extracción minera. }\end{array}$ & $\begin{array}{l}\text { El cumplimiento de lo establecido en la Cons- } \\
\text { titución. }\end{array}$ \\
\hline La inseguridad en las ciudades. & $\begin{array}{l}\text { Las situaciones que debe tener en cuenta la } \\
\text { política pública. }\end{array}$ \\
\hline La contaminación ambiental. & $\begin{array}{l}\text { Determinar las dimensiones involucradas en } \\
\text { una problemática social. }\end{array}$ \\
\hline Las ventas ambulantes. & Identificar los efectos de una medida. \\
\hline Habilidades que evalúa & \multicolumn{2}{|l|}{} \\
\hline Analizar modelos conceptuales y sus usos en decisiones sociales. \\
\hline Analizar los efectos en distintas dimensiones que tendría una posible intervención. \\
\hline Establecer relaciones que hay entre dimensiones presentes en una situación problemática. \\
\hline
\end{tabular}


terio de Educación, 2015), esta competencia evalúa dos aspectos: el reconocimiento de perspectivas y el nivel de confiabilidad de fuentes primarias y secundarias. En el primer caso se pretende que los estudiantes identifiquen y analicen perspectivas a través del examen de argumentos individuales y colectivos. En el segundo, que evalúen la validez de las fuentes a partir de su contextualización, y que establezcan su credibilidad, fiabilidad y los contextos de su producción y publicación. Esta competencia es central porque en ella se evalúan conceptos procedimentales relacionados con la lectura e interpretación de recursos como archivos históricos, material periodístico, información estadística, mapas, planos y fotografías. Además de interpretar información que contiene la fuente, los estudiantes deben establecer si hay argumentos sesgados, si la información es imparcial o por el contrario tiene alguna orientación hacia intereses específicos. En un país como el nuestro, donde la opinión pública es construida en parte por los medios de comunicación y donde estos tienen cada día más poder, es fundamental una competencia como esta, para determinar hasta qué punto los jóvenes desarrollan criterios para cuestionar la información proveniente de los medios, y concientizarlos sobre de la importancia de que ser ciudadanos informados y con criterios para determinar que los medios de comunicación pueden ser imparciales, pero también pueden estar al servicio de determinados intereses económicos y políticos.

En esta competencia se evalúa si los estudiantes están en capacidad de com- prender la realidad social desde una perspectiva sistémica como se ilustra en la tabla 5, a partir de la reconstrucción de la relación entre las dimensiones de una problemática social (Icfes y Ministerio de Educación, 2015). También se evalúa su habilidad para determinar la pertinencia de la solución que se le ha dado a una situación problemática determinada, los efectos de una intervención social y el impacto de la implementación de las políticas públicas. La prueba está centrada en la evaluación de conceptos, procedimientos y actitudes. En este sentido es congruente con los estándares de ciencias sociales cuando plantean que "existe la férrea convicción de que es necesario desarrollar las competencias de las y los estudiantes a partir de la conjugación de: (1) conceptos científicos, (2) metodologías y maneras de proceder científicamente y (3) compromiso social y personal" (Ministerio de Educación, 2006: p. 108).

Un cambio evidente en esta prueba en relación con la anterior es la relevancia del componente actitudinal. La fusión de las ciencias sociales con las competencias ciudadanas hace más evidente en toda la prueba, pero principalmente en la competencia de interpretación y análisis de perspectivas, lo que los estándares denominan la creación de espacios de reflexión para debatir asuntos polémicos y de aplicación de valores sociales a favor del interés público. Además, se observa fomento de un sentido crítico ante las actitudes y las relaciones dominantes que permitan tomar distancia respecto a los valores e ideologías establecidas. 


\section{Uso pedagógico de la prueba}

En términos pedagógicos, la prueba da a los maestros grandes posibilidades de trabajo. Producto de una investigación con profesores de ocho países de América Latina, Ravela (2010) determinó que las pruebas externas tienen una gran variedad de utilidades. Entre ellas destaca la toma de conciencia sobre contenidos que se deben trabajar con los alumnos, la apertura a nuevos enfoques conceptuales y prácticos, el acceso a instrumentos para el trabajo en el aula, inspiración y modelos para el diseño de materiales, el preparar a los estudiantes para las pruebas y el seguimiento de estudiantes individuales y de resultados grupales. De acuerdo con el campo del saber, sus necesidades y sus intereses, el profesor puede hacer énfasis en uno o en varios aspectos. Ejercicios como el presentado en la tabla 6 puede ser desarrollado por los maestros con el fin de utilizar la prueba para mejorar los aprendizajes de sus alumnos.

A diferencia de resolver cuestionarios cuando se acerca la presentación de las pruebas para que los estudiantes "aprendan a identificar las preguntas correctas", ejercicios como el ante-

\section{Tabla VI \\ Ejercicios para mejorar los aprendizajes}

(Fuente: Elaboración propia a partir de Icfes, Ministerio de Educación, 2014: p. 20)

\section{Pregunta del examen}

La acción de tutela es un mecanismo que busca la protección inmediata de los derechos fundamentales de los ciudadanos. Por tanto, esta se puede presentar ante un juez cuando

1. Se vulnera el derecho a la salud, porque está en riesgo la vida de la persona.

2. Alguien es expulsado de una agremiación, a pesar de estar al día en sus pagos.

3. Se busca proteger un derecho colectivo de constituir juntas de acción comunal.

4. Se legisla sobre el derecho a la vida y el acceso a un trabajo digno para las personas.

Usos pedagógicos que se le pueden dar a la prueba

\begin{tabular}{|l|l|l|}
\hline $\begin{array}{l}\text { Determinación } \\
\text { de contenidos }\end{array}$ & $\begin{array}{l}\text { Reflexión de las actividades } \\
\text { que tradicionalmente se } \\
\text { utilizan en la enseñanza }\end{array}$ & Revisión de las evaluaciones \\
\hline $\begin{array}{l}\text { Los derechos } \\
\text { fundamentales y } \\
\text { mecanismos de } \\
\text { reclamación de } \\
\text { derechos. }\end{array}$ & $\begin{array}{l}\text { Análisis de fallos te tutela } \\
\text { Entrevista a un juez } \\
\text { Ejercicios prácticos de diligen- }\end{array}$ & $\begin{array}{l}\text { Reflexión. El profesor se puede preguntar } \\
\text { si el tipo de preguntas que se hacen a los } \\
\text { estudiantes los lleva a determinar cuándo es } \\
\text { procedente uno u otro mecanismo de recla- } \\
\text { mación de derechos. } \\
\text { Reformulación de las preguntas que tradi- } \\
\text { cionalmente se hacen. }\end{array}$ \\
\hline
\end{tabular}


riormente planteado permiten que el maestro se provea de nuevos recursos para enseñar, que se cuestione sobre las actividades de aprendizaje que utiliza, que compare el nivel de dificultad y su forma de preguntar y que descubra o reconozca cuáles de las actividades de sus alumnos apuntan a la construcción de conceptos y al desarrollo de habilidades y cuáles no. Este tipo de ejercicios le sirven al profesor para hacer un seguimiento de los avances de sus estudiantes de manera pormenorizada, y emprender las acciones de mejora pertinentes. Dicho de otra manera, la prueba puede ser usada para definir contenidos, para determinar las habilidades por desarrollar en los estudian- tes y para cualificar las evaluaciones en actividades propias del maestro como el análisis de las Pruebas Saber, la planeación del diagnóstico de los estudiantes incluyendo las competencias y conocimientos evaluados en las pruebas, la determinación de fortalezas y dificultades de los estudiantes, el diseño de la estrategia pedagógica y el diseño e implementación de las evaluaciones incluyendo los componentes y las competencias que se evalúan.

El valor pedagógico de las pruebas está determinado también por la buena lectura que los docentes puedan hacer de los resultados obtenidos por sus alumnos y por la información que dichos resultados les suministren. En

\section{Cuadro I}

\section{Presentación de resultados en una prueba antes de 2014}

(Fuente: Instituto Colombiano para la Evaluación de la Educación, 2017. Recuperado desde http://www.icfesinteractivo.gov.co)

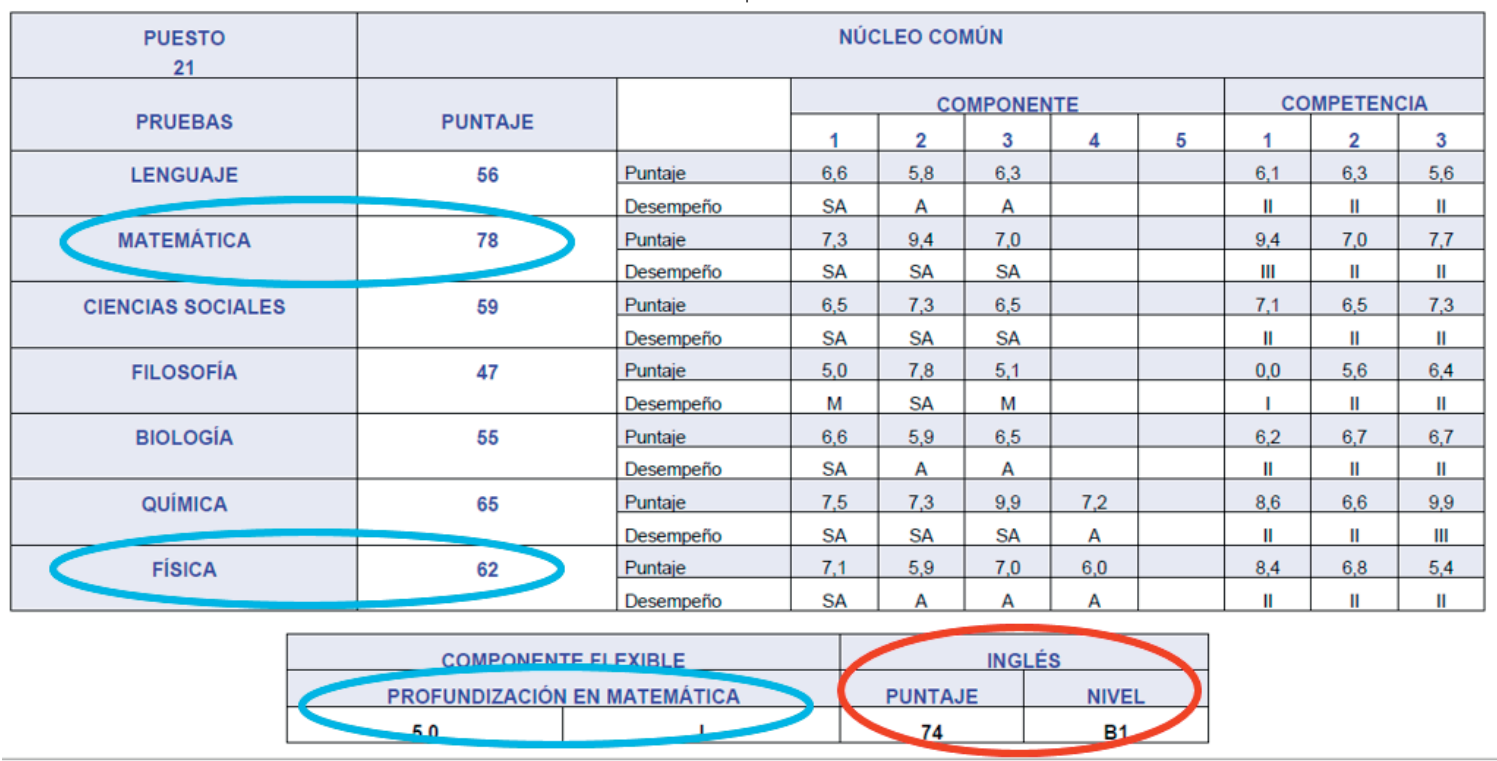




\section{Cuadro II \\ Reporte actual de resultados}

(Fuente: Instituto Colombiano para la Evaluación de la Educación, 2017.

Recuperado desde http://www.icfesinteractivo.gov.co)

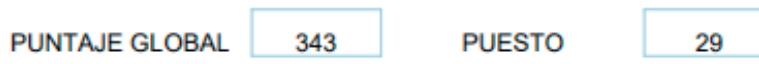

\begin{tabular}{|l|c|c|c|c|}
\multicolumn{1}{c}{ PRUEBA } & PUNTAJE & NIVEL & DECIL & \multicolumn{1}{c|}{$\begin{array}{c}\text { RANGO POBLACIONAL } \\
\text { PARA EL DECIL }\end{array}$} \\
\hline LECTURA CRITICA & 69 & & D10 & Entre $92.08 \%$ y $100 \%$ \\
\hline MATEMÁTICAS & 77 & & D10 & Entre $90.83 \%$ y $100 \%$ \\
\hline SOCIALES Y CIUDADANAS & 53 & & D06 & Entre $54.03 \%$ y $65.24 \%$ \\
\hline CIENCIAS NATURALES & 67 & & D10 & Entre $90.42 \%$ y $100 \%$ \\
\hline INGLÉS & 93 & B+ & D10 & Entre $90.87 \%$ y $100 \%$ \\
\hline
\end{tabular}

\begin{tabular}{|l|c|c|c|c|}
\hline \multicolumn{1}{|c|}{ SUB-PRUEBA } & PUNTAJE & NIVEL & DECIL & \multicolumn{2}{c|}{ RANGO POBLACIONAL } \\
\hline RAZONAMIENTO CUANTITATIVO & 74 & & D10 & Entre $91.28 \%$ y $100 \%$ \\
\hline COMPETENCIAS CIUDADANAS & 50 & & D05 & Entre $41.5 \%$ y $53.68 \%$ \\
\hline
\end{tabular}

este aspecto, se debe reconocer que las pruebas actuales perdieron mucho con relación a las anteriores; los resultados hasta 2014, como el presentado en el cuadro I, tenían una serie de detalles sobre los niveles de desempeño de los estudiantes que las nuevas hasta el momento no arrojan.

Nótese que en la prueba de ciencias sociales y en todas las demás se suministraba el promedio de la asignatura, la descripción numérica y cualitativa de cada uno de los niveles de desempeño por componentes (el espacio, el territorio, el ambiente y la población, el poder, la economía y las organizaciones sociales, el tiempo y las culturas) y la descripción numérica y cualitativa por competencias (interpretativa, argumentativa y propositiva). El análisis de esta información le permitía al profesor hacer una radiografía bastante completa del estado de sus alumnos y, lo más importante, tomar las medidas necesarias para mantener los buenos resultados y mejorar allí donde era necesario. Los resultados de la nueva prueba (véase el cuadro II) carecen de este tipo de información.

Como se evidencia en el cuadro II, la información del promedio del puntaje de la asignatura, y el rango de población para el decil no permiten mucho más allá de una comparación entre aéreas. Con el código que el Icfes le da a cada institución educativa, también 
los profesores y directivos pueden acceder al porcentaje de alumnos en cada uno de los niveles de desempeño de los promedios de cada prueba y la desviación estándar. Sin embargo, aunque esta información es importante porque permite determinar las fortalezas y las dificultades de los estudiantes en el área de una forma global y qué tan homogéneos o heterogéneos son, constituye una gran limitación que la nueva prueba no especifique los resultados por competencia, y por componente, porque como se ilustró, esos datos pueden guiar al maestro de una manera más precisa a establecer cuáles deben ser sus prioridades en el trabajo en el aula (Icfes, 2017). La información detallada, que en el examen anterior se suministraba al estudiante en sus resultados y en el actual ya no, puede ser obtenida mediante solicitud al Icfes, una vez se ha justificado el objetivo de la petición y se ha hecho un compromiso de uso académico de los resultados, pero el ideal sería que esos datos fueran de público conocimiento y de fácil acceso, principalmente para los maestros.

Como una necesidad propia y en cumplimiento de compromisos adquiridos en materia educativa con los demás países de la región, Colombia, al igual que otros países de América Latina, ha logrado consolidar un sistema de evaluación de la educación. Independientemente de las críticas que ha generado el modelo de educación por competencias, hoy el país cuenta con un conjunto de pruebas que evalúan los aprendizajes de los estudiantes de educación básica, media y superior. Si bien el Ministerio de Educación ha sido reiterativo en aclarar que la prueba no es el único indicador de la calidad de la educación, sí es evidente la importancia que dicho indicador ha tenido en los últimos años. Es también indiscutible que sus resultados son cada día más determinantes para la definición de la política educativa que adopta el Ministerio, las decisiones de las secretarías de Educación y el diseño e implementación de planes de mejoramiento de los colegios.

En la actualidad, las pruebas evalúan el nivel de desarrollo de competencias alcanzado por los estudiantes. Las críticas a dichas pruebas se han dado en varios sentidos. Para algunos, el Estado colombiano en su deseo de ingresar a la Organización para la Cooperación y el Desarrollo Económico (OCDE) y de cumplir con las exigencias de Banco Mundial y del Banco Interamericano de Desarrollo, implementa unas pruebas que no se compadecen con la inversión real que se ha hecho en la educación. Por otro lado, las críticas se enfocan en que una prueba nacional como la que se aplica desconoce la diversidad regional que caracteriza al país y las desigualdades económicas que determinan el ingreso y permanencia en la escuela de los niños y jóvenes colombianos. A estas críticas el Estado responde que las pruebas evalúan las competencias establecidas en los estándares curriculares, y estos contienen los conocimientos y habilidades básicas que todos los estudiantes del país deben tener, independiente de la región donde vivan o de su condición socioeconómica.

Una lectura reflexiva de la prueba de ciencias sociales nos permitió establecer que de un examen en el que se 
evaluaba principalmente la memorización de contenidos, se pasó a uno en el que se evalúa el desarrollo de competencias y luego se llegó a la estandarización de todas las pruebas Saber (3. ${ }^{\circ}$, $5 .^{\circ}, 9 .^{\circ}, 11 .^{\circ}$ y Pro. Esto persiguiendo el objetivo de que se evalúen las mismas competencias en diferentes grados de desarrollo. La estandarización o alineación logró que las condiciones de aplicación, el procesamiento de los resultados y las características técnicas del examen sean compartidos.

Lo anterior genera críticas relacionadas con que el examen se centre únicamente en las habilidades y conocimientos que se evalúan para el Informe del Programa Internacional para la Evaluación de Estudiantes (PISA por su sigla en inglés), o en aquellas que se consideren importantes en los organismos como la OCDE, el Banco Mundial y el Fondo Interamericano de Desarrollo, competencias que a los ojos de muchos pretenden profundizar y afianzar el modelo neoliberal, que pocos beneficios y condiciones de bienestar ha traído a las comunidades. El modelo de examen que hoy tenemos es para muchos la expresión y evidencia de la institucionalización en la escuela de unas políticas que vienen desde los centros de poder, y que están al servicio de sus intereses, pero que poco o nada llevan a que los estudiantes desarrollen unas habilidades y construyan unos conocimientos que los empoderen para disentir y revelarse precisamente contra las medidas que se toman en esos centros de poder y que día a día aumentan las condiciones de pobreza y desigualdad de la gran mayoría de la población.

\section{Consideraciones finales}

Las clasificaciones que se han hecho para pruebas estandarizadas (Wolfe, 2006; Ravela, 2006a), y el cuestionario de la prueba Saber $11^{\circ}$ en Colombia, nos indica que estamos ante un proceso de clasificación y certificación, en el cual se hace un reporte individual a cada uno de los evaluados. Este reporte incluye, además, una medición de competencias y de habilidades en campos de conocimiento específicos. La prueba Saber $11^{\circ}$ es un examen de acreditación que presentan los estudiantes colombianos de último año de la educación secundaria, que se convierte en requisito obligatorio para aquellos que quieren ingresar a la educación superior.

El impacto y las implicaciones de los resultados de estas pruebas ha ido en aumento en los últimos años, pues además de clasificar a los alumnos, clasifica a las instituciones las categorías, determina que estudiantes pueden acceder al programa de becas de educación superior que tiene el gobierno nacional, denominado "Ser pilo paga". Adicionalmente, es uno de los criterios de calidad que hace acreedores a incentivos económicos a maestros y directivos de las instituciones educativas públicas. Si bien, estas pruebas describen el logro de niveles particulares de desempeño en un área o en una asignatura (figura 2), nuestro planteamiento se centra en la necesidad de que estos resultados puedan ayudar a comprender aspectos curriculares y prácticas de enseñanza. Tal y cómo subraya (Wolfe, 2006) esto implica un desplazamiento de que preguntar cuánto sabe los estudiantes, a 
preguntar qué saben y qué son capaces de hacer. En la actualidad, si bien, podemos contar con una abundante producción escrita entorno a los sistemas nacionales de evaluación, referida a aspectos técnicos, instrumentales, y políticos de los mismo, todavía es muy débil la reflexión que se produce acerca de los factores relacionados con el aprendizaje (Perassi, 2008)

No estamos ante un asunto menor, pues desde el punto de (Ravela, 2006a), los críticos del énfasis creciente en los resultados de las pruebas argumentan que otorgar exceso de importancia solo algunas competencias y algunas habilidades distorsionan la educación, en tanto que otros objetivos importantes de la formación de los niños y jóvenes son ignorados porque no son evaluados en los exámenes. La gran importancia que se le está dando a los resultados de los exámenes estandarizados y no tanto a los procesos educativos conducentes a mejores aprendizajes, puede llevar a que se enseñen las cosas específicas y necesarias para pasar las pruebas, en lugar de dominios de conocimientos más generales. En el peor de los casos, la enseñanza se puede orientar a contestar mecánicamente un examen y a identificar claves para detectar la respuesta correcta. En palabras de Ravela (2006a), el puntaje de la prueba y el grado de comprensión no son lo mismo; se requiere de una generalización, y es la generalización, no la prueba en sí misma, lo que importa debido a que "cuando se enseña para responder el tipo de ítem específicos de las pruebas, la validez de la inferencia sobre el nivel de logro de los estudiantes se ve amenazada" (p.194).

De allí, la importancia de las reflexiones como las de Esquivel (2006) sobre necesidad de que las pruebas incluyan preguntas de respuesta o final abierto, producción de ensayos, solución de problemas, producción de materiales o discursos para exhibición pública, elaboración de documentos. Por su parte Wolff (2006) resalta la importancia de que los profesores se vinculen a los procesos evaluación de la calidad de la educación desde el comienzo, y no sean unos simples usuarios de sus resultados si se desea que dichas evaluaciones tengan impacto en la educación. En esta misma línea argumentativa Ravela (2006b) sugiere que si la apuesta es a que los sistemas de evaluación aporten información relevante para revisar y mejorar las prácticas pedagógicas, deberíamos preguntarnos en qué grado la información que se ofrece sobre los resultados de las pruebas estandarizadas resultan pertinente y enriquecedores para comprender los procesos de aprendizaje de los alumnos y para analizar las estrategias de trabajo en el aula.

\section{Referencias bibliográficas}

CASTILLO, M. (2004). La evaluación por competencias y sus implicaciones pedagógicas 1991-2004. Revista Enunciación, $\mathrm{n}^{\circ} 9$ (1), 68-78.

ESQUIVEL, J.M. (2006). El diseño de las pruebas para medir el logro académico: ¿Referencia a normas o a criterios? En P. Arregui (Ed.), Sobre 
estándares y evaluaciones en América Latina (82-97).Santiago de Chile: PREAL -Editorial San Marino.

MINISTERIO DE EDUCACIÓN NACIONAL DE COLOMBIA. (1994). Ley 115 o Ley General de Educación. Bogotá.

MINISTERIO DE EDUCACIÓN NACIONAL DE COLOMBIA. (2002). Lineamientos curriculares de ciencias sociales. Bogotá.

MINISTERIO DE EDUCACIÓN NACIONAL DE COLOMBIA. (2006). Estándares básicos de competencias en lenguaje, matemáticas, ciencias y ciudadanas. Lo que los estudiantes deben saber y saber hacer con lo que aprenden. Bogotá.

INSTITUTO COLOMBIANO PARA EL FOMENTO DE LA EDUCACIÓN SUPERIOR. (2004). Evaluación de las pruebas de Estado. Bogotá: Cooperativa Editorial Magisterio.

INSTITUTO COLOMBIANO PARA LA EVALUACIÓN DE LA EDUCACIÓN Y MINISTERIO DE EDUCACIÓN NACIONAL. (2011). Examen de Estado. Interpretación de los resultados. Bogotá.

INSTITUTO COLOMBIANO PARA LA EVALUACIÓN DE LA EDUCACIÓN Y MINISTERIO DE EDUCACIÓN NACIONAL. (2013). Sistema de evaluación y estandarización. Alineación del examen Saber $11^{\circ}$. Bogotá.

INSTITUTO COLOMBIANO PARA LA EVALUACIÓN DE LA EDUCACIÓN Y MINISTERIO DE EDUCACIÓN
NACIONAL. (2015). Guía de interpretación y uso de resultados de las Pruebas Saber 11. ${ }^{\circ}$. Versión 1. Bogotá. INSTITUTO COLOMBIANO PARA LA EVALUACIÓN DE LA EDUCACIÓN Y MINISTERIO DE EDUCACIÓN NACIONAL. (2016). Guía de interpretación y uso de resultados de las Pruebas Saber 11. . Establecimientos educativos. Versión 1. Bogotá

INSTITUTO COLOMBIANO PARA LA EVALUACIÓN DE LA EDUCACIÓN. (2017). Informe de los resultados de la prueba SABER 11. Guía de interpretación y uso de resultados de las Pruebas Saber $11^{\circ}$. Versión 1. Recuperado el 15 de febrero de 2017 desde http://www.icfesinteractivoresultados.gov.co.

PERASSI. Z. (2008). La evaluación en educación: un campo de controversias. San Luis: Ediciones LAE. Laboratorio de alternativas educativas.

PÉREZ, V. (2015). Del examen a la evaluación pasando por el paradigma del emprendedor. Evaluar ¿para qué?, ¿para quiénes? Revista Con-ciencia social: anuario de didáctica de la geografía, la historia y las ciencias sociales, 19, 145-152.

PRADO, V., y MEDINA, A. (2014). Características de la enseñanza y la evaluación de los aprendizajes en educación básica primaria. Papeles, 11, 20-35. [Recuperado el 25 de febrero de 2017] de http://csifesvr.uan.edu.co/ index.php/papeles/article/view/394 
RAVELA, P. (2006a). ¿Cómo presentan sus resultados los sistemas nacionales de evaluación educativa en América Latina? En P. Arregui (Ed.), Sobre estándares y evaluaciones en América Latina (181-248). Santiago de Chile: PREAL -Editorial San Marino.

RAVELA, P. (2006b). Los resultados de las evaluaciones educativas en la prensa. En P. Arregui (Ed.), Sobre estándares y evaluaciones en América Latina (295-384). Santiago de Chile: PREAL -Editorial San Marino.

RAVELA, P. (2010). ¿Qué pueden aportar las evaluaciones estandarizadas a la evaluación en el aula? Programa de Promoción de la Reforma Educativa en América Latina y el Caribe. Preal, CINDE.Serie Documentos, $\mathrm{N}^{\circ}$ 473-25. Recuperado el 4 febrero de 2017 desde https://www.academia.edu/1414652/_ Qu\%C3\%A9_pueden_aportar_las_ evaluaciones_estandarizadas_a_la_ evaluaci\%C3\%B3n_en_el_aula

ROJAS, R. (2014). Competencias en lenguaje: relaciones entre la escuela y las pruebas de Estado. Estudios sobre la calidad de la educación en Colombia. Bogotá: Instituto Colombiano para la Evaluación de la Educación.

SOUTO, X.M., FUSTER, C. y SÁIZ, J. (2014) Un camino de ida y vuelta: reválidas y selectividad en las rutinas escolares de la enseñanza de Geografía e Historia. En J. Pagés y A. Santisteban (Eds.), Una mirada al pasado y un proyecto de futuro Investigación e innovación en didáctica de las ciencias sociales (pp.157-165). Barcelona:
AUPDCS y Universitat Autònoma de Barcelona.

VALVERDE, G. (2006). La interpretación justificada y el uso apropiado de los resultados de las mediaciones. En P. Arregui (Ed.), Sobre estándares y evaluaciones en América Latina (7081). Santiago de Chile: PREAL -Editorial San Marino.

WOLFE, R. (2006). El dilema de las granularidad en el diseño del sistema de evaluación: cobertura curricular VS. Cobertura poblacional. En P. Arregui (Ed.), Sobre estándares y evaluaciones en América Latina (61-69).Santiago de Chile: PREAL -Editorial San Marino.

WOLFF, L. (2006). Las evaluaciones educacionales en América Latina: Avance actual y futuros desafíos. En P. Arregui (Ed.), Sobre estándares y evaluaciones en América Latina (1352).Santiago de Chile: PREAL -Editorial San Marino. 